\title{
Recent results of the second generation of vector vortex coronagraphs on the high-contrast imaging testbed at JPL
}

\author{
Dimitri Mawet ${ }^{a, b}$, Eugene Serabyn ${ }^{b},{\text { Dwight } \text { Moody }^{b}, \text { Brian Kern }^{b} \text {, Albert Niessner }}^{b}$, Andreas \\ Kuhnert $^{b}$, David Shemo ${ }^{c}$, Russell Chipman ${ }^{d}$, Stephen $\mathrm{McClain}^{d}$, and John Trauger ${ }^{b}$ \\ ${ }^{a}$ European Southern Observatory, Alonso de Córdova 3107, Vitacura 7630355, Santiago, Chile; \\ ${ }^{b}$ Jet Propulsion Laboratory - California Institute of Technology, 4800 Oak Grove Dr, \\ Pasadena, CA 91109, USA; \\ ${ }^{c}$ JDSU, 2789 Northpoint Parkway, Santa Rosa, CA 95407, USA; \\ ${ }^{d}$ University of Arizona, 1630 E University Blvd., Tucson, AZ 85721, USA
}

\begin{abstract}
The Vector Vortex Coronagraph (VVC) is an attractive internal coronagraph solution to image and characterize exoplanets. It provides four key pillars on which efficient high contrast imaging instruments can be built for ground- and space-based telescopes: small inner working angle, high throughput, clear off-axis discovery space, and simple layout. We present the status of the VVC technology development supported by NASA. We will review recent results of the optical tests of the second-generation topological charge $4 \mathrm{VVC}$ on the actively corrected High Contrast Imaging Testbed (HCIT) at the Jet Propulsion Laboratory (JPL). New VVC contrast records have been established.
\end{abstract}

Keywords: High contrast imaging, exoplanets, phase-mask coronagraphy, vector vortex coronagraph, liquid crystal polymers, wavefront control, electric field conjugation

\section{INTRODUCTION}

This paper describes the setup, device, and results of the test of a second-generation topological charge 4 vector vortex coronagraph (VVC) on the high contrast imaging testbed (HCIT) at JPL. The VVC, described in details in Ref. 1 (these proceedings), is a transparent phase-based coronagraph allowing access to very small inner working angles (IWA) which is an appealing property, especially when considering a limited-size space-based telescope dedicated to extra-solar planetary system imaging and characterization. The VVC also provides a high throughput and a clear $360^{\circ}$ off-axis discovery space. One of its remarkable features is its simplicity, and compatibility with a Lyot coronagraph layout.

The VVC is one possible realization of the vortex coronagraph. ${ }^{2,3}$ The phase ramp of the vortex is generated by a circularly symmetric halfwave plate that applies two conjugated helical phase structures to the orthogonal circular polarization states of the incoming natural light. It has been demonstrated many times ${ }^{2,4-6}$ that such a phase structure multiplied to the point spread function (PSF) of an unobscured circular pupil leads downstream to a redirection of the light outside the geometric image of the input pupil. A Lyot-plane aperture (the Lyot stop) then blocks all of the diffracted starlight. Light from off-axis objects misses the center of the vortex and propagates normally.

\section{GENERATION 2 VECTOR VORTEX CORONAGRAPH}

In Ref. 1 (these proceedings), we review the principles and technologies of the vector vortex coronagraph family (many different types of VVC exist, adapted to different purposes). The particular device tested here is of topological charge 4 , meaning that the vortex phase ramp $e^{i \phi}$, with $\phi=4 \theta$, where $\theta$ is the focal plane azimuthal coordinate, spans 4 waves $(8 \pi)$. While trading-off IWA, which becomes $1.7 \lambda / d$ instead of $0.9 \lambda / d$ for a topological charge 2 vortex, increasing the topological charge allows reducing the sensitivity of contrast to low-order wavefront

Further author information: send correspondence to dmawet@eso.org

Techniques and Instrumentation for Detection of Exoplanets $\mathrm{V}$, edited by Stuart Shaklan,

Proc. of SPIE Vol. 8151, 81511D - @ 2011 SPIE · CCC code: 0277-786X/11/\$18 · doi: 10.1117/12.896070

Proc. of SPIE Vol. 8151 81511D-1 


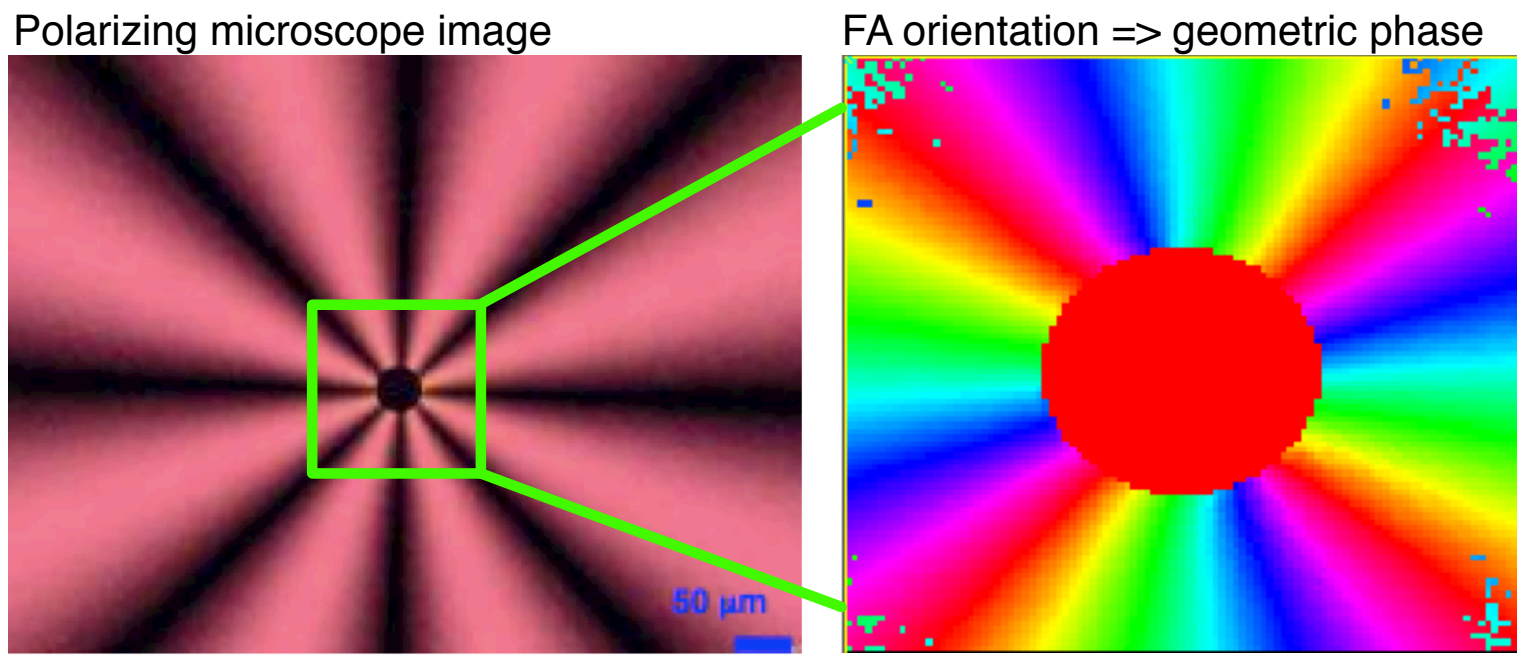

Figure 1. Left: actual device under a polarizing microscope (crossed polarizer). While the device is totally transparent, its vectorial nature, or in other word, its polarization manipulation nature, appears in this very simple test (useful for quick diagnostics), transforming a polarization map into an intensity map. The central opaque spot $40 \mu \mathrm{m}$ in diameter is easily visible. Right: zoom on the central part using a high resolution polarimetric microscope from the Polarization Laboratory of the College of Optical Sciences (University of Arizona), showing the fast axis orientation rotating about the center. Here, this rotation yields a geometric phase change equals to $4 \theta$, where $\theta$ is the focal plane azimuthal coordinate, subsequently spanning 4 waves or $8 \pi$.

errors, which is sometimes a necessary feature. ${ }^{7}$ Indeed, slowly evolving thermal and mechanical constraints affect the optical train, and subsequently the wavefront stability mostly at large spatial scales. These low-order aberrations in turn degrade the contrast at small angles. Designing the system to meet the stability requirements necessary to reach and maintain a very high contrast at these angles is very demanding and costly, hence the tradeoff between IWA and sensitivity to low-order aberrations.

The second-generation topological charge 4 device was specifically manufactured for HCIT tests by JDSU in 2009 (see Fig. 1), following the promising test of the first generation of charge 4 VVC in $2008 .{ }^{8}$ The same liquid crystal polymer technology (LCP, see Ref. 1, these proceedings) was used but the process was optimized to answer identified weaknesses of the gen-1 prototype. Indeed, the VVC tested in 2008 performed well (best contrast result of $\sim 10^{-7}$ ) but was limited by several factors, ${ }^{8}$ summarized here:

- Ghosts originating from multiple reflections inside the multi-layer structure of the sample.

- Diffraction due to the central opaque spot covering the so-called region of disorientation, ${ }^{3}$ where the LCP rod-like molecules are not able to follow the fast axis spin at the vortex center $(d=40 \mu \mathrm{m}$ for the gen-1 charge $4 \mathrm{VVC})$.

- Chromatic leakage of the single LCP layer VVC, subject to the natural dispersion of the LCP birefringence.

We started with the optimization of the internal reflections within the complex multi-layer stack of the actual VVC device. Without going into details, it is important to realize that, while the vortex function is implemented by a single layer of LCP, the actual device is fact composed of about 8 layers of different materials, including a pair of substrates sandwiching the LCP layer itself for protection, optical adhesives, the precursor layer, ${ }^{1}$ and the AR coatings on both external faces. The index-matching optimization was straightforward and consisted in choosing the appropriate material for each layer (including a new substrate material), as well as improved broadband AR coatings. All in all, the new design attenuates the ghosts by two orders of magnitudes compared to the first generation. 
The gen-2 VVC is equipped with a central $40 \mu \mathrm{m}$ spot similar to the gen-1 mask. To temporarily short-circuit the limitation associated to the size of the central spot/defect while true technical solutions are being investigated to shrink it, we chose to exploit the recent reconfiguration of the HCIT to accommodate a new $64 x 64$ Xinetics DM (as opposed to the $32 \times 32$ used thus far, see below), that would allow increasing the focal ratio $F=f / d$ of the beam while keeping a reasonable number of actuators thanks to the bigger, denser DM. Increasing the $F$ increases the resolution element size with respect to the opaque mask covering the vortex center, and thus significantly reducing its perturbing influence on the VVC operation.

Finally, as discussed in Ref. 1, we are currently exploring two routes to mitigate the chromatic leakage harmful effect. However, the gen-2 mask is still a single-layer LCP VVC, meaning that it is chromatic and only capable of delivering its best contrast performance over a limited wavelength range, designed to be $\sim 800 \mathrm{~nm}$. One of the two identified chromaticism mitigation methods, known as chromatic leakage filtering ${ }^{*}$, could be tested on the HCIT, simply using polarizers, inserted upstream and downstream from the VVC. Fig. 2 and Fig. 3 presents the HCIT layout and the location of the inserted polarizing elements, with which we carried out the latter approach (see Sect. 5).

Despite a design wavelength of $800 \mathrm{~nm}$, the manufacturer JDSU measured a halfwave $\lambda=814 \mathrm{~nm}$ over a region of several mm, corresponding to the resolution of the Axoscan polarimeter used for the measurement. Using an optimized high-resolution microscope polarimeter, the Polarization Laboratory of the College of Optical Sciences (University of Arizona) independently measured the halfwave $\lambda=806 \mathrm{~nm}$ over a $1 \mathrm{~mm}^{2}$ area centered on the vortex. We performed our own measurement on the HCIT, in coronagraphic mode, i.e. with the PSF centered on the vortex. The spatial scale of the measurement is therefore the resolution element $(F \lambda \approx 40-80$ $\mu \mathrm{m})$, which is the one that matters. We mapped the chromatic leakage as a function of wavelength, and arrived to a minimum at $793 \mathrm{~nm}$. While all around $800 \mathrm{~nm}$, the design wavelength, these different measurements are explained by the different scales employed and the apparent non-uniformity of the LCP layer.

\section{LABORATORY SETUP: THE HCIT}

The JPL high contrast imaging testbed (HCIT) is a state-of-the-art coronagraphic bench (see Ref. 11 for details). Using a reflective scheme, this facility highlights a vacuum chamber mounted on vibration isolation blocks, and high-order deformable mirrors (DM) to perform accurate wavefront control. Two types of DM are currently available: a new superpolished 64x64 Xinetics mirror, and a series of 32x32 Xinetics mirrors.

The HCIT available light sources are provided by a suite of lasers $(633 \mathrm{~nm}, 785 \mathrm{~nm}, 809 \mathrm{~nm}$, etc.) and a supercontinuum laser, filtered by one of six passband filters. Five filters have center wavelengths of 768,784 , 800,816 , and $832 \mathrm{~nm}$ and (nearly square) $16 \mathrm{~nm}$ bandpasses $(\Delta \lambda / \lambda=2 \%)$. Two broadband filters are centered at $800 \mathrm{~nm}$ and have passband of $80 \mathrm{~nm}(10 \%)$ and $160 \mathrm{~nm}(20 \%)$, respectively. Inside the tank, the light from the source is passed through an unresolved pinhole $(\mathrm{d}=5 \mu \mathrm{m}$, compared to $\mathrm{F} \lambda=20 \mu \mathrm{m})$, effectively acting as a point-source, simulating a star.

When power is applied to the DM, the phase errors of the system are dominated (at the spatial frequencies of interest to us) by the surface of the DM, whose actuators have differing voltage-to-displacement relationships. After powering up the DM, the first activity is to flatten the system, using a modified Gerchberg-Saxton estimation procedure. The goal of this initialization step is to produce a front-end exit pupil with a uniform phase across the aperture, where front-end refers to the optics preceding the occulter. This technique typically leaves a $5 \mathrm{~nm}$ rms residual front-end wavefront error.

At periodic intervals during normal operation, the occulter is re-centered on the image of the source. Remotely controlled actuators translate the occulting mask to compensate slow environmental drifts. The photometric calibration is also periodically recalculated. The photometric calibration scheme used relates images taken with

\footnotetext{
${ }^{*}$ The method is reviewed in Ref. 1 (these proceedings), and presented in more details in Ref. 1,7,9,10. In short, the VVC phase ramp is purely geometrical hence intrinsically achromatic. The dispersion of the LCP retardance generates a pupil leakage uniform in amplitude, bearing no phase modification. Owing to the vectorial nature of the VVC, the chromatic leakage is orthogonal to the main geometrical achromatic vortex term in the circular polarization basis. This property suggests that a pair of crossed circular polarizer-analyzer sandwiching the VVC can filter out the chromatic leakage while letting any off-axis object through.
} 


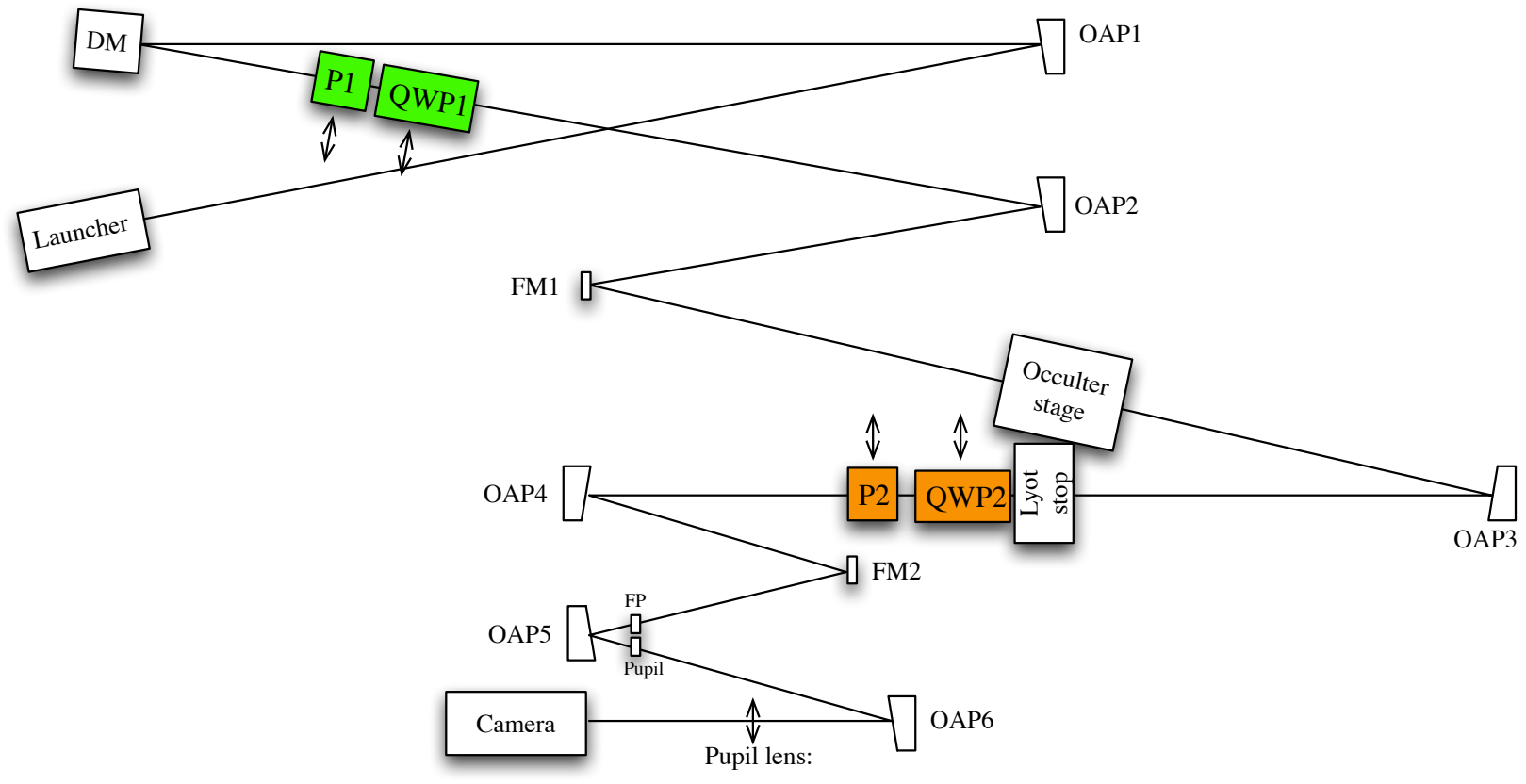

Figure 2. Layout of the HCIT showing the approximate location of the optical elements and the chief ray. The circular polarizers-analyzer (combination of a polarizer $\mathrm{P}$ and quarterwave plate, QWP) are on translation stages and can be taken in and out. The coronagraph mount is on a 3-axis translation stage. The Lyot stop wheel can also be adjusted in $\mathrm{x}$. The camera travels in $\mathrm{z}$ for fine focus adjustments and phase retrieval.
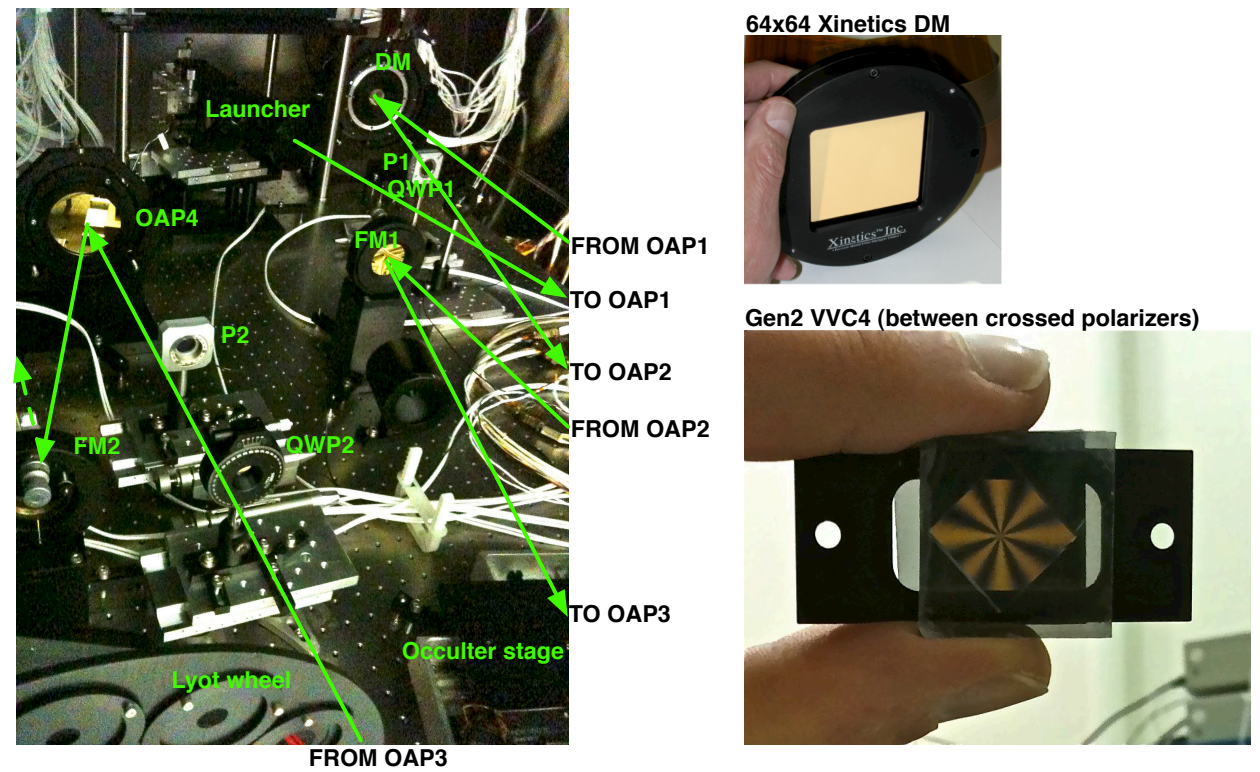

Figure 3. Left: partial picture of the HCIT showing the critical elements of the experiments. The DM, the polarizers P1 and P2, quarterwave plates QWP1, and QWP2, constituting the circular polarizer and analyzer, respectively. Top right: picture of the Xinetics 64x64 DM. Bottom right: picture of the generation $2 \mathrm{VVC}$ of topological charge 4 seen between crossed polarizers after its optical tests on the HCIT.

the occulter in-place to images taken with the occulter out-of-place, establishing a ratio between the light at the peak of the unocculted PSF to the wings of the occulted PSF (well beyond the region in the image directly 
Table 1. Key setup parameters for both experiments (monochromatic and broadband).

\begin{tabular}{|c|c|c|c|c|c|c|}
\hline Test & $\lambda$ & Entrance pupil diam & $F$ & Lyot stop & IWA-OWA & Contrast \\
\hline Monochromatic $\left(^{*}\right)$ & $785 \mathrm{~nm}$ & $32 \mathrm{~mm}$ & 47 & $85 \%$ & $2.5 \lambda / d-12 \lambda / d$ & $3.5 \times 10^{-9}$ \\
\hline Broadband $(* *)$ & $760-840 \mathrm{~nm}$ & $19 \mathrm{~mm}$ & 79 & $92 \%$ & $2.5 \lambda / d-7 \lambda / d$ & $2.6 \times 10^{-8}$ \\
\hline
\end{tabular}

(*) Without chromatic leakage filtering.

$(* *)$ With chromatic leakage filtering.

controllable to the Nyquist spatial frequency of DM actuators). This ratio has been seen empirically to be constant at the 10-20\% level (depending on DM voltage settings) and stable (for similar DM voltages) to a few percent, (few $\%$ variations over months), and is used to calibrate photometrically every image taken during normal operation.

To create a so-called dark hole, i.e. a region of the image where the wavefront correction provided by the DM is applied, we used the electric field conjugation algorithm, or EFC. ${ }^{12}$ The name of the algorithm comes from the fact that the DMs actuators are set so as to superpose the negative of the electric field onto the image plane, conceptually making the image intensity zero. The algorithm is divided into two parts, estimation and correction. The complex electric field amplitude in the image plane is estimated, then the estimate is processed to determine a correction to be applied to the DM actuators. These two parts are functionally independent, and each could be used separately with other approaches. Each EFC iteration begins with a DM setting which is the result of the prior iterations correction result (or is the flat setting, to begin the first iteration).

The vectorial nature of the VVC, which applies two conjugated phase ramps to the two orthogonal circular components of the incoming unpolarized natural light, imposes the use of a polarizing beam splitter just before the camera in order to disentangle both components. One or both transfer functions can be incorporated to the EFC correction scheme. Here we worked with a single one.

\section{MONOCHROMATIC TEST AT 785 NM}

The first test we conducted used a $785 \mathrm{~nm}$ laser source, and a $32 \mathrm{~mm}$ diameter entrance pupil (see Table 1). In this configuration, the $F \approx 47$, making the central $40-\mu \mathrm{m}$ spot appear about 1 resolution element across (note that 1 resolution element $=9$ camera pixels), or $0.5 F \lambda$ in radius. The wavefront was flattened using the procedure described earlier, and the EFC loop started.

EFC requires an accurate optical model of the system. We used our knowledge of the DM a priori calibrated with interferometry, and all the information we have on the VVC itself, including the central spot effect. The control region was defined as a half circle appearing left of the image of the source, starting at $2 \lambda / d$ and going all the way up to $12 \lambda / d$ (see Fig. 4).

After tweaking the control loop parameters (regularization), EFC converged fast ( $\sim 10-20$ iterations) to deep contrast levels, down to $3.5 \times 10^{-9}$ median contrast over the dark hole region (Fig. 4). We defined the inner working angle at $2.5 \lambda / d$, where we measured a contrast of $\sim 10^{-8}$. As mentioned before, the theoretical IWA of the $\mathrm{VVC}$ of topological charge 4 is $1.7 \lambda / d$, leaving room for improvement.

\section{POLYCHROMATIC TEST RESULTS}

We inserted the polarizing elements as shown in Fig. 2 and Fig. 3 to attempt the first demonstration of the chromatic leakage filtering method with wavefront control ${ }^{\dagger}$. Unfortunately the size of the polarizing elements was smaller than the nominal entrance pupil size of $32 \mathrm{~mm}$. Stopping down the beam to $\sim 20 \mathrm{~mm}$ forced us to use fewer DM actuators, which subsequently reduced the size of the dark hole region (see Table 1). Note that the shape of the dark hole can be square or circular.

\footnotetext{
${ }^{\dagger}$ Note that this technique was already successfully demonstrated in the lab with several "geometrical phase-mask coronagraphs" at the Hokkaido University, ${ }^{10,13,14}$ including a VVC made out of photonic crystals, but without wavefront control.
} 


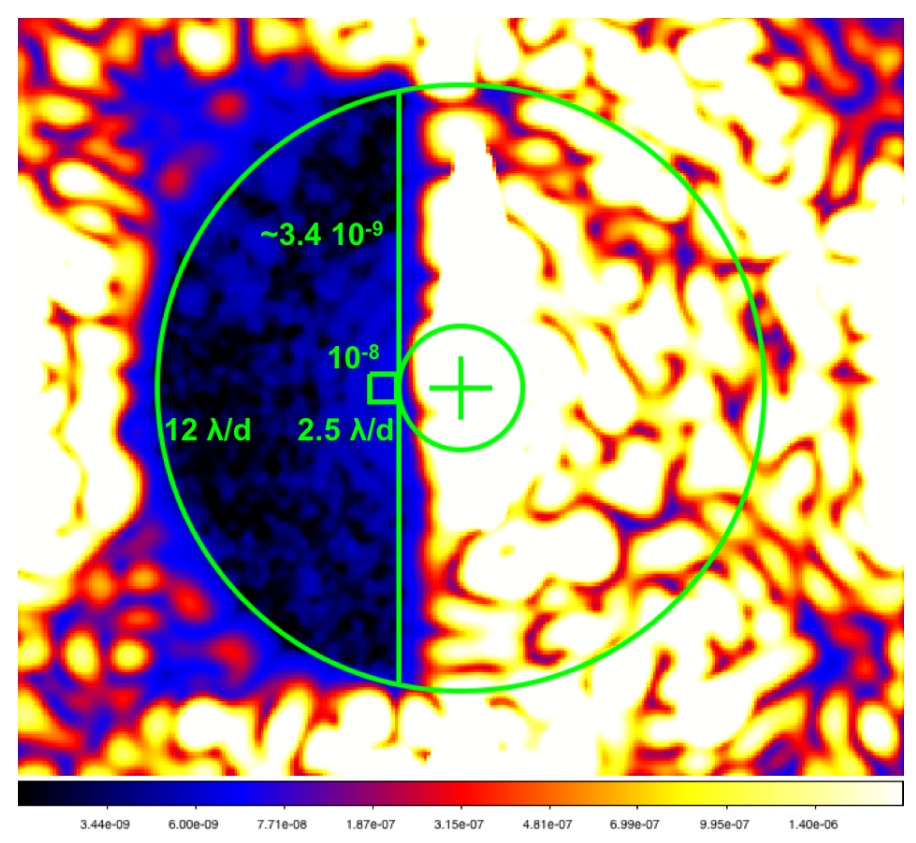

Figure 4. Dark hole generated at $785 \mathrm{~nm}$ with the second-generation VVC or topological charge 4 on the HCIT. The median contrast between 2.5 and $12 \lambda / d$ is $\sim 3.4 \times 10^{-9}$.

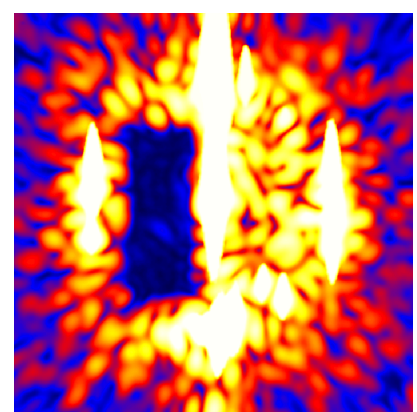

$760-840 \mathrm{~nm}$

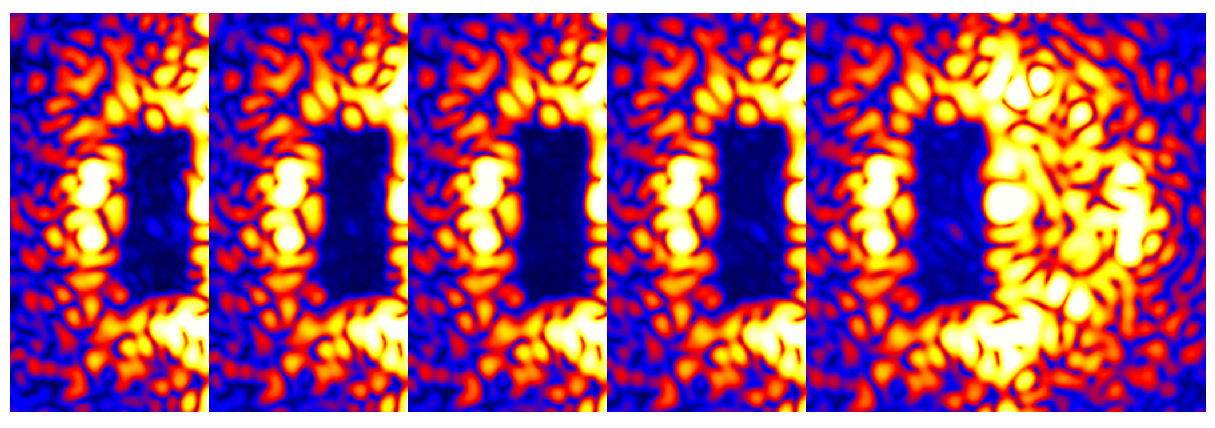

$760-778 \mathrm{~nm} \quad 778-792 \mathrm{~nm} \quad 792-808 \mathrm{~nm} \quad 808-824 \mathrm{~nm} \quad 824-840 \mathrm{~nm}$

Figure 5. Left: Laboratory demonstration of VVC contrast averaging $\sim 2.5 \times 10^{-8}$ across a $760-840 \mathrm{~nm}(10 \%)$ spectral bandwidth. The dark field extends from a vertical line offset $2.5 \lambda / d$ left of the occulted "star" to an outer radius at $7 \lambda / d$ centered on the "star". Right: Contrast images in five contiguous $2 \%$ passbands shows the evolution of the background field of speckles across the $10 \%$ bandwidth image at left.

We changed the prescription of the EFC model to reflect the hardware changes: the beam size and relative diameter of the VVC spot being the major changes we made. The control was done at the wavelength closest to the measured halfwave wavelength of $793 \mathrm{~nm}$ and at the center of the $10 \%$ passband filter, i.e. $800 \mathrm{~nm}$. We then scanned through the $2 \%$ filters available around $800 \mathrm{~nm}$, as well as used the $10 \%$ filters for a true broadband contrast check. The results of the broadband experiment are shown in Fig. 5 and Fig. 6. Let us emphasize the fact that no "broadband wavefront control" per se was performed, i.e. no feedback from outside of the $800 \mathrm{~nm}$ passband was used to drive the wavefront control. Indeed, the EFC energy minimization algorithm was used at $800 \mathrm{~nm}$ only, and the other wavelength passively measured. The next step will be to perform true broadband wavefront control, minimizing the energy at all wavelengths simultaneously, such as reported in Ref. 12,15.

\section{DISCUSSION}

While the prototype used for this test run was designed to work at a single wavelength, measured to be $793 \mathrm{~nm}$, it performed exceptionally well outside of its comfort zone. 


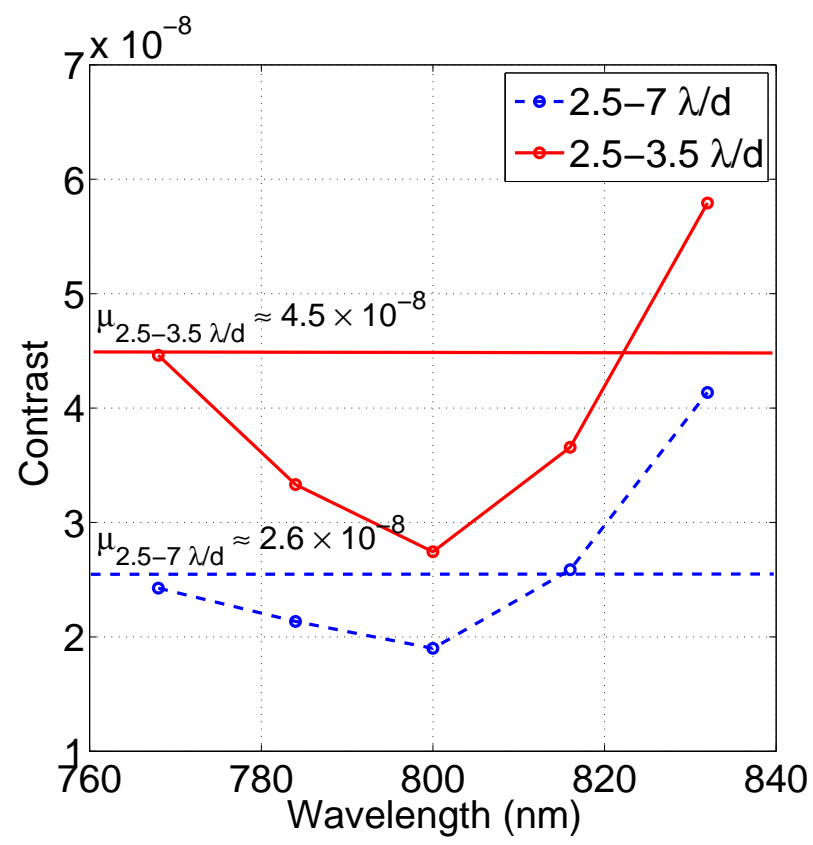

Figure 6 . Contrast results across the $10 \%$ bandwidth measured in 5 contiguous $2 \%$ filters. The contrast is measured close in $(2.5-3.5 \lambda / d)$ and inside the whole dark hole extending in this polychromatic test from 2.5 to $7 \lambda / d$. Note that the contrast within the true $10 \%$ filter is consistent with the averaged contrast measured in the $52 \%$ filters.

First of all, the monochromatic test was performed at $785 \mathrm{~nm}$, which should have resulted in a degraded contrast by at least of factor of 10 worse than actually measured. The reason for the excellent $785 \mathrm{~nm}$ result can be explained by the wavefront control and the coherence of the input source, that allowed nulling the residual down to the $3.5 \times 10^{-9}$ level. The floor we reached was characterized as being incoherent light largely resulting from scattering in the system, accounting for at least $1.5 \times 10^{-9}$. Other sources of limitation can include: backend polarizing element quality, mask imperfections (dust, non-uniformities, effect from the central opaque spot, and other cosmetic defects).

Second, the polychromatic tests using the chromatic leakage filtering method, performed surprisingly well, showing broadband contrast gains up to 2 orders of magnitude above the intrinsic limitation of the single-layer VVC prototype. This test was the first time chromatic leakage filtering was applied to a vectorial coronagraph together with wavefront control. It can then be considered as a spectacular proof of concept.

\section{CONCLUSIONS}

After the very first test in $2008,{ }^{8}$ this second pass using an improved second-generation VVC of topological charge 4 achieved a two orders of magnitude gain in contrast both monochromatically and over a $10 \%$ bandpass ${ }^{\ddagger}$. We expect the third and subsequent generations of devices in the making to be intrinsically achromatic over $20 \%$ bandwidth (thanks to a 3-layer design, see Ref. 1,7). Improved baffling and better polarizers should make the contrast level deeper by an order of magnitude or two, bringing the VVC down to the levels required by Earthlike planet detection and characterization. We demonstrated inner working angle of $2.5 \lambda / d$, which is close to but still not at the theoretical limit of $1.7 \lambda / d$ for a topological charge $4 \mathrm{VVC}$, leaving some margin for further progress in that area as well. As the VVC technologies evolve even further (see Ref. 1, these proceedings), the VVC coronagraph family becomes an appealing practical solution to a small space-based telescope dedicated to exoplanet imaging and characterization (see Ref. 16, these proceedings).

\footnotetext{
${ }^{\ddagger}$ Note the last result presented in Ref. 16, these proceedings: a contrast of $\sim 4 \times 10^{-8}$ was measured in a $20 \%$ bandwidth, using the chromatic leakage filtering method and the device presented in this paper.
} 


\section{ACKNOWLEDGMENTS}

This work was carried out at the European Southern Observatory (ESO) site of Vitacura (Santiago, Chile), and the Jet Propulsion Laboratory (JPL), California Institute of Technology (Caltech), under contract with the National Aeronautics and Space Administration (NASA).

\section{REFERENCES}

[1] Mawet, D. and al., "Taking the vector vortex coronagraph to the next level for ground- and space-based exoplanet imaging instruments: review of technology developments in the USA, Japan, and Europe," in [Society of Photo-Optical Instrumentation Engineers (SPIE) Conference Series], Society of Photo-Optical Instrumentation Engineers (SPIE) Conference Series (2011).

[2] Mawet, D., Riaud, P., Absil, O., and Surdej, J., "Annular Groove Phase Mask Coronagraph," ApJ 633, 1191-1200 (Nov. 2005).

[3] Mawet, D., Serabyn, E., Liewer, K., Hanot, C., McEldowney, S., Shemo, D., and O’Brien, N., "Optical Vectorial Vortex Coronagraphs using Liquid Crystal Polymers: theory, manufacturing and laboratory demonstration," Optics Express 17, 1902-1918 (Feb. 2009).

[4] Foo, G., Palacios, D. M., and Swartzlander, Jr., G. A., "Optical vortex coronagraph," Optics Letters 30, 3308-3310 (Dec. 2005).

[5] Jenkins, C., "Optical vortex coronagraphs on ground-based telescopes," MNRAS 384, 515-524 (Feb. 2008).

[6] Swartzlander, Jr., G. A., "The optical vortex coronagraph," Journal of Optics A: Pure and Applied Optics 11, 094022-+ (Sept. 2009).

[7] Mawet, D., Pueyo, L., Moody, D., Krist, J., and Serabyn, E., "The Vector Vortex Coronagraph: sensitivity to central obscuration, low-order aberrations, chromaticism, and polarization," in [Society of Photo-Optical Instrumentation Engineers (SPIE) Conference Series], Society of Photo-Optical Instrumentation Engineers (SPIE) Conference Series $\mathbf{7 7 3 9}$ (July 2010).

[8] Mawet, D., Trauger, J. T., Serabyn, E., Moody, Jr., D. C., Liewer, K. M., Krist, J. E., Shemo, D. M., and O'Brien, N. A., "Vector vortex coronagraph: first results in the visible," in [Society of Photo-Optical Instrumentation Engineers (SPIE) Conference Series], Society of Photo-Optical Instrumentation Engineers (SPIE) Conference Series $\mathbf{7 4 4 0}$ (Aug. 2009).

[9] Mawet, D., "Annular Groove Phase Mask: An Achromatic Vortex Coronagraph Intended at Differential Polarimetric Imaging," in [In the Spirit of Bernard Lyot: The Direct Detection of Planets and Circumstellar Disks in the 21st Century], (June 2007).

[10] Murakami, N., Uemura, R., Baba, N., Nishikawa, J., Tamura, M., Hashimoto, N., and Abe, L., "An EightOctant Phase-Mask Coronagraph," PASP 120, 1112-1118 (Oct. 2008).

[11] Trauger, J. T. and Traub, W. A., "A laboratory demonstration of the capability to image an Earth-like extrasolar planet," Nature 446, 771-773 (Apr. 2007).

[12] Give'on, A., Kern, B., Shaklan, S., Moody, D. C., and Pueyo, L., "Broadband wavefront correction algorithm for high-contrast imaging systems," in [Society of Photo-Optical Instrumentation Engineers (SPIE) Conference Series], Society of Photo-Optical Instrumentation Engineers (SPIE) Conference Series 6691 (Sept. 2007).

[13] Murakami, N., Nishikawa, J., Yokochi, K., Tamura, M., Baba, N., and Abe, L., "Achromatic Eight-octant Phase-mask Coronagraph using Photonic Crystal," ApJ 714, 772-777 (May 2010).

[14] Murakami, N., Baba, N., Ise, A., Sakamoto, M., and Oka, K., "Laboratory demonstration of an optical vortex mask coronagraph using photonic crystal," in [In the Spirit of Lyot 2010], (Oct. 2010).

[15] Moody, D. C., Gordon, B. L., and Trauger, J. T., "Design and demonstration of hybrid Lyot coronagraph masks for improved spectral bandwidth and throughput," in [Society of Photo-Optical Instrumentation Engineers (SPIE) Conference Series], Society of Photo-Optical Instrumentation Engineers (SPIE) Conference Series 7010 (Aug. 2008).

[16] Serabyn, E., Mawet, D., and Wallace, J., "Recent progress in vector vortex coronagraphy ," in [Society of Photo-Optical Instrumentation Engineers (SPIE) Conference Series], Society of Photo-Optical Instrumentation Engineers (SPIE) Conference Series (2011). 